SCIENTIFIC LETTER

\title{
Coronary collateral flow and peripheral blood monocyte concentration in patients treated with granulocyte- macrophage colony stimulating factor
}

\author{
R Zbinden, R Vogel, B Meier, C Seiler
}

Heart 2004;90:945-946. doi: 10.1136/hrt.2003.018259

$\mathrm{E}$ stablished options for revascularisation of coronary artery disease (CAD) are angioplasty or bypass surgery, both of which are unsuitable in about one in five patients because of the severity of atherosclerosis, comorbidities, or both. An alternative therapeutic option is to promote the endogenous development of collateral vessels.

There are three principle ways in which vessel growth can occur: (1) vasculogenesis-this occurs primarily during embryonic development by differentiation of haematopoietic stem cells; (2) angiogenesis-this is the sprouting of new vessels out of existing ones, occurring during embryonic development and under certain conditions in the adult; (3) arteriogenesis-pre-existing collateral arterioles transform into arteries by dilatation, smooth muscle cells and endothelial cells proliferate, and consecutively acquire a typical arterial structure. ${ }^{1}$ Angiogenesis is induced by various cytokines, is dependant on ischaemia, and mainly results in high resistance capillaries; true collateral artery growth is temporally and spatially dissociated from ischaemia and results in large interconnecting arterioles which are required for the salvage of myocardium. ${ }^{2}$

Aside from their role in defending the host against invading pathogens, monocytes play an important role in building collateral arteries during arteriogenesis. Arteriogenesis is initiated by increased shear forces in pre-existing arterioles (due to an occlusion or narrowing of the main vessel) with subsequent upregulation of adhesion molecules and cytokine production by endothelial cells. Circulating monocytes adhere and migrate into deeper parts of the vessel wall and stimulate vessel growth by releasing cytokines, growth factors, and enzymes such as metalloproteinases. ${ }^{3}$

Previous studies, in a model of acute ligation of the femoral artery in mice and rabbits, showed stimulation of arteriogenesis by increased concentrations of blood monocytes and inhibition of collateral artery growth with selective depletion of peripheral blood monocytes. The negative effect was reversed when monocyte depletion was compensated for by an injection of purified monocytes. ${ }^{4}$

The purpose of this study was to elucidate whether there is an association between the amount of collateral flow change in response to granulocyte-macrophage colony stimulating factor (GM-CSF) treatment ${ }^{5}$ and blood monocyte concentration.

\section{METHODS}

Twenty one patients (mean (SD) age 75 (10) years, 10 men and 11 women) with extensive CAD not eligible for, or unwilling to undergo, coronary artery bypass surgery, and with at least one stenotic lesion suitable for percutaneous coronary intervention (PCI), were included in the study. Patients were randomly assigned to a two week, double blind protocol of intracoronary followed by subcutaneous GM-CSF (Molgramostim) $(\mathrm{n}=10)$ or placebo $(\mathrm{n}=11)$. Collateral flow index (CFI) was assessed invasively during balloon

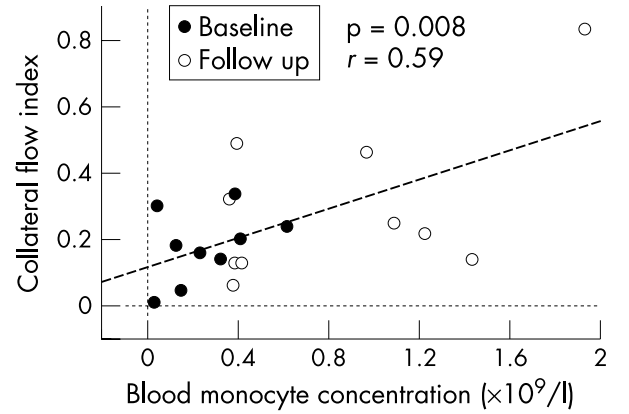

Figure 1 Regression plot of patients receiving GM-CSF (baseline and follow up measurements).

occlusion at inclusion and after two weeks. CFI was determined by simultaneous measurement of mean aortic pressure $\left(\mathrm{P}_{\mathrm{ao}}\right)$ obtained via the guiding catheter, distal coronary artery pressure during balloon occlusion $\left(\mathrm{P}_{\mathrm{occl}}\right)$ obtained via a 0.014 inch pressure monitoring guidewire, and central venous pressure (CVP). CFI was calculated as $\left(\mathrm{P}_{\mathrm{occl}}-\right.$ CVP) divided by $\left(\mathrm{P}_{\mathrm{ao}}-\mathrm{CVP}\right)$.

A complete white blood cell count of all patients at inclusion and at follow up was performed.

\section{RESULTS}

Mean (SD) increase in total leucocytes $\left(7.23(6.63) \times 10^{9} / \mathrm{l}\right.$, $\mathrm{p}=0.007)$, neutrophils $\left(6.25(6.24) \times 10^{9} / \mathrm{l}, \mathrm{p}=0.01\right)$, and eosinophils $\left(1.03(1.29) \times 10^{9} / \mathrm{l}, \mathrm{p}=0.04\right)$ was significant after GM-CSF treatment; increases in monocytes $(0.45$ $\left.(0.68) \times 10^{9} / \mathrm{l}, \mathrm{p}=0.079\right)$, lymphocytes $\left(0.16(0.80) \times 10^{9} / \mathrm{l}\right.$, $\mathrm{p}=\mathrm{NS})$, and basophils $\left(0.01(0.08) \times 10^{9} / \mathrm{l}, \mathrm{p}=\mathrm{NS}\right) \mathrm{did}$ not reach significance. There was a significant correlation between peripheral blood monocyte concentration and invasively assessed CFI (fig 1). There was no association between the increase in CFI and total peripheral leucocyte, granulocyte, or lymphocyte concentration.

There was no correlation between peripheral monocyte count and CFI in the placebo group.

\section{DISCUSSION}

Heil and colleagues showed, in an ischaemic hindlimb model in rabbits and mice, the critical role of monocytes in collateral vessel development. ${ }^{4}$ Our study demonstrates a direct association between peripheral blood monocyte count in humans treated with GM-CSF and invasively assessed CFI. This underlines the importance of monocytes and their

Abbreviations: $C A D$, coronary artery disease; $C F I$, collateral flow index; CVP, central venous pressure; GM-CSF, granulocytemacrophage colony stimulating factor; $\mathrm{PCl}$, percutaneous coronary intervention 
inflammatory response in the vessel wall during arteriogenesis because GM-CSF prolongs survival of monocytes/macrophages and protects a high number of attracted monocytes from apoptosis, therefore enhancing their arteriogenic potential. Additionally, the recruitment of monocytes from bone marrow, which increases the number in peripheral blood, seems to be important. Conversely, there is no association between increased total leucocyte, lymphocyte or granulocyte count, although the number of these cells is equally elevated after GM-CSF treatment; this would indicate a minor involvement of these cells in arteriogenesis or no involvement at all.

\section{Authors' affiliations}

R Zbinden, R Vogel, B Meier, C Seiler, Department of Cardiology,

University Hospital Bern, Switzerland
Correspondence to: Professor Christian Seiler, University Hospital Bern, Freiburgstrasse Bern, CH-3010, Switzerland; christian.seiler@insel.ch

Accepted 28 November 2003

\section{REFERENCES}

1 Carmeliet P. Mechanisms of angiogenesis and arteriogenesis. Nat Med 2000;6:389-95.

2 Buschmann I, Schaper W. Arteriogenesis versus angiogenesis: two mechanisms of vessel growth. News Physiol Sci 1999;14:121-5.

3 Arras $M$, Ito WD, Scholz D, et al. Monocyte activation in angiogenesis and collateral growth in the rabbit hindlimb. J Clin Invest 1998;101:40-50.

4 Heil M, Ziegelhoeffer T, Pipp F, et al. Blood monocyte concentration is critical for the enhancement of collateral artery growth (arteriogenesis). Am J Physiol Heart Circ Physiol 2002;3:H241 1-9.

5 Seiler C, Pohl T, Wustmann K, et al. Promotion of collateral growth by granulocyte-macrophage colony-stimulating factor in patients with coronary artery disease: a randomized, double-blind, placebo-controlled study. Circulation 2001;104:2012-7.

\section{IMAGES IN CARDIOLOGY}

\section{Left main coronary artery occlusion in a patient with solitary coronary ostium in the right aortic sinus}

\begin{abstract}
A n 82 year old woman with a history of high blood pressure and chronic atrial fibrillation without oral anticoagulation was admitted to the emergency department because of a syncopal episode followed by chest pain. Until that day she was totally asymptomatic.

On admission the patient was in cardiogenic shock; the ECG showed ST segment elevation from V4-V6, DI, and aVL. An urgent coronary artery angiogram was performed. An intra-aortic balloon counterpulsation was inserted. The first contrast injection was done in the right coronary ostium (panel A). The right coronary artery (RCA) was normal without any obstruction. During the same injection, a vessel arising from the proximal RCA was seen $\left({ }^{*}\right)$. Initial suspicion was an anomalous origin of left circumflex coronary artery (LCX), the most common coronary artery anomaly. The left coronary ostium was impossible to cannulate from the left aortic sinus, and aortography was performed (panel B; Ao, aorta): there was no left coronary ostium, suggesting that the vessel arising from the proximal RCA $\left({ }^{*}\right)$ was the left coronary artery. Two angioplasty guide wires (panel C) crossed the obstruction point $(\dagger)$. Balloon angioplasty was performed at the left main coronary artery (LMCA) bifurcation, displaying the whole coronary artery tree from a single injection in the right coronary artery ostium (panel D; LAD, left anterior descending coronary artery)
\end{abstract}
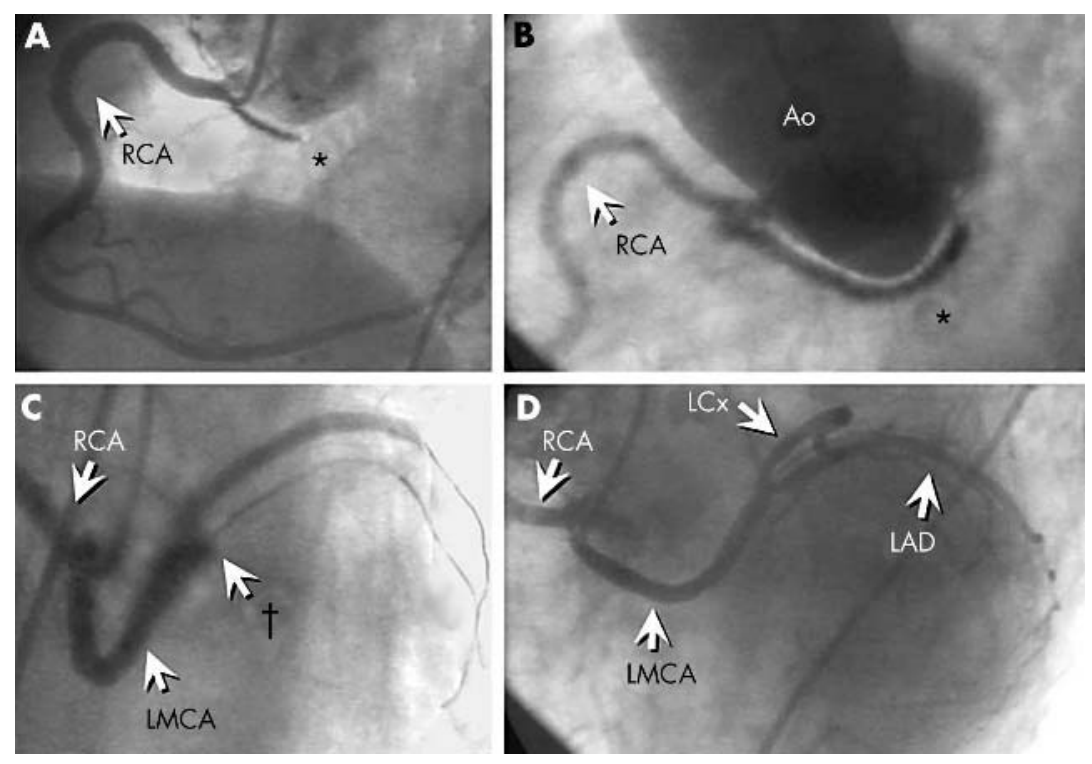

Anomalous LMCA takes an aberrant retroaortic course, as seen in panels A and B, which shows a left anterior oblique projection, where the LMCA goes backwards. This kind of anomalous coronary artery distribution is the most uncommon type.

B Ibanez

M Cordoba

J Farre

bibanez@telefonica.net 